\title{
COORDENAÇÃO MOTORA FINA DE ESCOLARES COM DISLEXIA E TRANSTORNO DO DÉFICIT DE ATENÇÃO E HIPERATIVIDADE
}

\author{
Fine motor coordination of students with dyslexia \\ and attention deficit disorder with hiperactivity
}

\author{
Paola Matiko Martins Okuda ${ }^{(1)}$, Maria Dalva Lourencetti( ${ }^{(2)}$, Lara Cristina Antunes dos Santos ${ }^{(3)}$, \\ Niura Aparecida de Moura Ribeiro Padula(4), Simone Aparecida Capellini ${ }^{(5)}$
}

\begin{abstract}
RESUMO
Objetivos: descrever e comparar o desempenho da coordenação motora fina em escolares com dislexia e com transtorno do déficit de atenção e hiperatividade utilizando parâmetros de desempenho motor e idade cronológica da Escala de Desenvolvimento Motor. Método: participaram 22 escolares do ensino fundamental, de ambos os gêneros, na faixa etária de 6 a 11 anos de idade distribuídos em: Gl: 11 escolares com transtorno do déficit de atenção e hiperatividade e Gll: $11 \mathrm{com}$ dislexia. Como procedimento, provas de motricidade fina da Escala de Desenvolvimento Motor foram aplicadas. Resultados: os resultados revelaram diferença estatisticamente significante entre a idade motora fina e a idade cronológica de GI e Gll. Conforme a classificação da Escala do Desenvolvimento Motor, $90 \%$ dos escolares de GI e Gll apresentaram desenvolvimento motor fino muito inferior ao esperado para a idade e $10 \%$ dos escolares com dislexia apresentam desenvolvimento normal baixo ao esperado para a idade e $10 \%$ dos escolares com transtorno do déficit de atenção e hiperatividade apresentaram desenvolvimento inferior ao esperado para a idade. Conclusão: concluímos que tanto os escolares com dislexia como os com TDAH deste estudo apresentam atrasos na coordenação motora fina, demonstrando que os participantes desta pesquisa apresentam dificuldades em atividades que exijam destreza, quadro característico do transtorno do desenvolvimento da coordenação. Estudos complementares estão sendo conduzidos pelos autores deste estudo para poder verificar e comprovar se o perfil motor fino dos escolares encontrados neste estudo se assemelham ou se diferem de acordo com o quadro apresentado pelos mesmos.
\end{abstract}

DESCRITORES: Destreza Motora; Dislexia; Transtorno da Falta de Atenção com Hiperatividade

(1) Terapeuta Ocupacional voluntária do Grupo de Pesquisa $\mathrm{CNPq}$ "Linguagem Aprendizagem e Escolaridade" da Faculdade de Filosofia e Ciências da Universidade estadual Paulista - FFC/UNESP - Marília - SP; Aluna especial do Programa de pós-graduação em Educação (Mestrado) da Faculdade de Filosofia e Ciências da Universidade estadual Paulista - FFC/UNESP, Marília, SP.

(2) Neuropsicóloga do Ambulatório de Desvios da Aprendizagem do HC/FM/UNESP - Botucatu, SP; Mestre em Saúde Coletiva pela Universidade do Sagrado Coração - USC, Bauru, SP.

(3) Neurologista Infantil; Responsável pelo Ambulatório de Desvios da Aprendizagem do HC/FM/UNESP, Botucatu, SP.

(4) Neurologista Infantil; Doutora em Ciências Médicas pela FCM/UNICAMP, Campinas, SP; Docente do departamento de Neurologia e Psiquiatria da FM/UNESP, Botucatu, SP.

(5) Fonoaudióloga; Docente do departamento de Fonoaudiologia e do programa de pós-graduação da Faculdade de Filosofia e Ciências da Universidade Estadual Paulista - FFC/ UNESP, Marília, SP; Doutora e Pós-doutora em Ciências Médicas pela FCM/UNICAMP, Campinas, SP.

Conflito de interesses: inexistente

\section{INTRODUÇÃO}

Durante anos, o desenvolvimento motor e o cognitivo foram estudados separadamente, como se houvesse pouca relação entre eles. Contudo, estudos recentes têm demonstrado que a motricidade e a cognição estão muito mais relacionadas do que se pensava, principalmente na etapa de alfabetização ${ }^{1,2}$. O período da alfabetização é o momento em que há uma superposição de habilidades para a ocorrência da aprendizagem da leitura e escrita. Essa aprendizagem envolve habilidades cognitivas, linguísticas e motoras que exigem dos escolares o uso dos componentes sensório-motores e perceptivos, ou seja, a capacidade de decodificação das palavras e a ação motora adequada para a execução do ato motor da escrita ${ }^{3}$. 
Segundo Schirmer et al. ${ }^{4}$, o processo de aquisição da linguagem escrita, assim como o da linguagem oral, envolve diversas regiões cerebrais, entre elas a área parieto-occipital. Na região occipital, o córtex visual primário é o responsável pelo processamento dos símbolos gráficos, e as áreas do lobo parietal são responsáveis pelas questões visuo-espaciais da grafia. Essas informações processadas são reconhecidas e decodificadas na área de Werneck, responsável pela compreensão da linguagem, e a expressão da linguagem escrita necessita da ativação do córtex motor primário e da área de Broca. Para todo este processo ocorrer, é importante que as fibras de associação intrahemisféricas estejam intactas.Na região anterior do cérebro é que acontece o planejamento, organização e execução do movimento. Outras áreas também participam da ação motora, enviando mensagens, dosando a força, a agilidade, fornecendo feedback visual, táctil e auditivo, permitindo, dessa forma, o ajuste constante do movimento ${ }^{5}$. Desta forma, qualquer alteração neurofuncional nas regiões cerebrais responsáveis pela entrada, processamento e execução da informação pode comprometer o desempenho práxico-produtivo envolvido no ato de ler e escrever.

Entre os transtornos que acometem o desempenho práxico-produtivo envolvido no ato de ler e escrever estão a dislexia e o Transtorno do Déficit de Atenção e Hiperatividade $(T D A H)^{6-12}$. A literatura $^{13-16}$ aponta que tanto escolares com dislexia como escolares com TDAH podem apresentar, em sua história acadêmica, o fracasso escolar, quer seja determinado por alterações na entrada da informação, como ocorre no TDAH, ou no processamento cognitivo da leitura, como ocorre na dislexia.

Estudos demonstraram que tanto a população disléxica como a população com TDAH apresentam dificuldades na coordenação bimanual, destreza manual e habilidades motoras finas, o que justificaria a ocorrência da disgrafia nesta população ${ }^{3,16-18}$. Entretanto, apesar de estes estudos apontarem essas dificuldades, a literatura especializada não referiu se as dificuldades motoras finas e a disgrafia encontrada nestas populações são partes dos quadros de dislexia e de TDAH ou se é decorrente de um quadro de transtorno do desenvolvimento da coordenação (TDC) em comorbidade.

As alterações motoras são comumente conhecidas como Transtorno do desenvolvimento da coordenação motora (TDC), que é descrito como um comprometimento nas habilidades motoras, sendo encontrado entre $5 \%$ e $7 \%$ de crianças em idade escolar na população geral ${ }^{12,19}$. Os sinais do TDC são geralmente associados a desajeitamento e inconsistência no desempenho de tarefas, coordenação motora pobre, problemas de ritmo e sua transferência de aprendizagem, declínio do desempenho com a repetição, tensão corporal e excesso de atividade muscular em tarefas motoras, podendo apresentar dificuldades nas tarefas de auto-cuidado, como vestir-se, nas atividades acadêmicas, de lazer e esportes, além de problemas com interações sociais ${ }^{19}$.

A literatura especializada refere que, ao menos, $50 \%$ dos escolares com problemas de aprendizagem são identificados concomitantemente com uma desordem no desenvolvimento da coordenação motora ${ }^{20,21}$. Na presença de dificuldades de aprendizagem, há maior probabilidade das funções práxicas e gnósicas estarem alteradas comprometendo a destreza, a velocidade de manipulação de objetos, exatidão do movimento, a postura da mão e as habilidades de escrita e consequentemente as tarefas funcionais, como abotoar, usar tesoura, manusear moedas, lápis e escrever ${ }^{17,22}$.

No Brasil, ainda são escassos os estudos sobre o TDC e, desta forma, conforme aponta a literatura nacional ${ }^{14,15}$, é possível que escolares que apresentam alterações motoras associadas à dislexia e ao TDAH sejam sub-diagnosticados acarretando o desenvolvimento de programas de intervenção psicoeducacionais inadequados para esta população. Ainda, apesar de existirem na literatura internacional ${ }^{23-26}$ e nacional ${ }^{27,28}$ baterias que se propõem a avaliar a função motora fina, não há a descrição de normativa para a população brasileira, assim, para a realização deste estudo, optou-se pelo uso das provas de motricidade fina da Escala de Desenvolvimento Motor $^{28}$ devido à Escala apresentar resultados normativos para a população brasileira que permitem o estabelecimento da comparação do desempenho motor e a idade cronológica dos escolares deste estudo consoante os parâmetros da escala.

Com base no exposto, este estudo tem por objetivos descrever e comparar o desempenho da coordenação motora fina em escolares com dislexia e escolares com transtorno do déficit de atenção e hiperatividade utilizando os parâmetros de desempenho motor e idade cronológica da Escala de Desenvolvimento Motor.

\section{MÉTODO}

Participaram deste estudo 22 escolares na faixa etária de 6 anos e 3 meses a 11 anos de idade, de ambos os gêneros, com nível socioeconômico médio, do ensino fundamental de escolas públicas e privadas. A classificação do nível socioeconômico foi realizada com base no estudo estatístico 
do Índice de Desenvolvimento Socioeconômico - IDESE ${ }^{29}$, garantindo assim, a homogeneidade da amostra do ponto de vista socioeconômico. Os escolares foram divididos em dois grupos:

- Grupo I(GI): 11 escolares com diagnóstico interdisciplinar de Transtorno do déficit de atenção e hiperatividade (TDAH), sendo $11(100 \%)$ do gênero masculino. Esses escolares não apresentaram intercorrências pré, peri e pós-natais, nem tampouco atraso no desenvolvimento neuropsicomotor, apresentando, portanto, o TDAH de origem primária.

- Grupo II (GII): 11 escolares com diagnóstico interdisciplinar de dislexia, sendo $4(36 \%)$ escolares do gênero feminino e 7 (64\%) do gênero masculino. Estes escolares não apresentaram intercorrências pré, peri e pós-natais, nem tampouco atraso no desenvolvimento neuropsicomotor. Todos os escolares apresentaram atraso no desenvolvimento da linguagem e transtorno fonológico presente na linguagem oral e escrita, apresentando, portanto, a dislexia de origem primária.

Os escolares dos grupos citados foram selecionados a partir do diagnóstico interdisciplinar confirmado de dislexia e TDAH, obtido a partir de avaliação neurológica, neuropsicológica, fonoaudiológica e terapêutico ocupacional em 65 escolares encaminhados para o Ambulatório de Neurologia Infantil - Desvios da Aprendizagem do Hospital das Clínicas da Faculdade de Medicina- FM/UNESP Botucatu-SP no período de maio a junho de 2009.

Para a realização deste estudo, foram utilizados os seguintes procedimentos:

- Termo de Consentimento: Conforme resolução do Conselho Nacional de Saúde CNS 196/96, anteriormente ao início das avaliações, os pais ou responsáveis dos pacientes selecionados assinaram o termo de Consentimento Pós-Informado para autorização da realização do estudo.

- Provas de motricidade fina da Escala de Desenvolvimento Motor (EDM $\left.{ }^{28}\right)$ : A EDM compreende um conjunto de provas muito diversificadas e de dificuldades graduadas conforme a idade, conduzindo a uma exploração minuciosa de diferentes habilidades motoras do desenvolvimento da criança. Entretanto, para este estudo, foram utilizadas somente as provas específicas para avaliar a coordenação motora fina (MF). Foram aplicadas as seguintes provas de motricidade fina: Cubos em Torre, Cubos em Ponte, Agulha, Nó, Labirinto, Bolinha de Papel, Ponta do polegar, Lançar uma Bola, Círculo com polegar e Agarrar uma Bola. Os materiais utilizados para a aplicação deste procedimento foram: bola, cubos, cesto com buraco e pedaços de papel, conforme descrito no manual de aplicação do procedimento.

Os testes foram realizados segundo os critérios descritos no manual do próprio autor, por uma terapeuta ocupacional, em uma sala de atendimento do Ambulatório de Neurologia Infantil - Desvios da aprendizagem do Hospital das Clínicas da Faculdade de Medicina- FM/UNESP - Botucatu-SP, em uma única sessão, com duração média de trinta minutos.

Os resultados foram analisados seguindo os critérios definidos na $\mathrm{EDM}^{28}$, ou seja, por meio do cálculo do quociente motor fino (QMF). Esse cálculo foi realizado por meio da divisão da idade motora fina (IMF) pela idade cronológica (IC) e multiplicado por cem (QMF= IMF/IC x 100), resultando em uma pontuação referente à função motora fina. Com a obtenção desta pontuação, esta foi classificada de acordo com parâmetros estabelecidos na Tabela 1.

Tabela 1 - Classificação da escala de desenvolvimento motor (Rosa Neto, 1996)

\begin{tabular}{cc}
\hline Pontuação & Classificação \\
\hline 130 ou mais & Muito Superior \\
$120-129$ & Superior \\
$110-119$ & Normal Alto \\
$90-109$ & Normal Médio \\
$80-89$ & Normal Baixo \\
$70-79$ & Inferior \\
69 ou menos & Muito Inferior \\
\hline
\end{tabular}

Fonte: Escala do Desenvolvimento Motor (Rosa Neto, 1996)

Depois do término deste estudo, todos os pais ou responsáveis pelos escolares foram informados sobre os resultados por meio de relatórios e orientados sobre a necessidade do desenvolvimento de atividades com a função motora fina tanto em casa como em sala de aula. Para esta orientação foram entregue duas cópias do manual de atividades de função motora ${ }^{30}$, sendo uma para os pais ou responsáveis e uma para o professor destes escolares. Aos escolares que se encontravam em terapia multidisciplinar, um destes manuais também foi entregue para os terapeutas.

Este trabalho foi aprovado pelo Comitê de Ética em Pesquisa da Faculdade de Filosofia e Ciências da Universidade Estadual Paulista - FFC/UNESP/ Marília - SP, sob o protocolo de número 3405/2006.

Os resultados obtidos foram analisados estatisticamente por meio do Teste dos Postos Sinalizados de Wilcoxon, com a finalidade de verificar possíveis diferenças entre a idade cronológica 
dos escolares e a idade da função motora; Teste de Mann-Whitney, com o objetivo de verificar diferença entre os grupos deste estudo; Teste de Friedman para verificar possíveis diferenças de desempenho dos grupos nos subtestes de função motora fina quando comparadas concomitantemente; e Análise de Correlação de Spearman, com o objetivo de verificar o grau de relacionamento entre os subtestes de função motora fina. O nível de significância (valor de p) adotado foi de 5\% $(0,050)$ e está marcado com asterisco. Para a análise dos dados, foi utilizado o programa SPSS (Statistical Package for Social Sciences), em sua versão 13.0.

\section{RESULTADOS}

Na Tabela 2, pode-se observar que os resultados dos subtestes de função motora fina, analisada pelo Teste Friedman, revelaram diferença estatisticamente significante tanto para o Gl como para o Gll, indicando que ocorreu diferença de desempenho entre os escolares com dislexia e TDAH nos subtestes de função motora fina.

Como na Tabela 2 foi evidenciada diferença estatisticamente significante para todos os subtestes, foi aplicado o Teste dos Postos Sinalizados de Wilcoxon para verificar em qual subteste os escolares do Gl e Gll apresentaram melhor desempenho (Tabela 3).

Na Tabela 3, pôde-se observar que os escolares do $\mathrm{Gl}$ apresentaram desempenho superior em construção de cubos em torre (CT) se comparado a labirinto (Lab), bolinha de papel (BP), ponta do polegar (PP), lançar uma bola (LB) e círculo com o polegar (Cpo). Apresentaram desempenho superior na construção de cubos em ponte (CP) se comparado

Tabela 2 - Distribuição da média, desvio padrão e valor de p dos grupos GI e Gll nos subtestes de função motora fina da escala do desenvolvimento motor

\begin{tabular}{cccccc}
\hline Grupo & Subtestes & Média & Desvio- padrão & Mediana & Valor de $\mathbf{p}$ \\
\hline & CT & 1,00 & 0,00 & 1,00 & \\
CP & 1,00 & 0,00 & 1,00 & \\
& Agu & 1,00 & 0,00 & 1,00 & \\
Nó & 0,91 & 0,30 & 1,00 & \\
& Lab & 0,09 & 0,30 & 0,00 & $<0,001^{*}$ \\
& BP & 0,45 & 0,47 & 0,50 & \\
& PP & 0,41 & 0,38 & 0,50 & \\
& LB & 0,36 & 0,45 & 0,00 & \\
& Cpo & 0,41 & 0,38 & 0,50 & \\
& AB & 0,77 & 0,41 & 1,00 & \\
\hline & CT & 1,00 & 0,00 & 1,00 & \\
& CP & 1,00 & 0,00 & 1,00 & \\
& Agu & 1,00 & 0,00 & 1,00 & \\
& Nó & 0,82 & 0,40 & 1,00 & \\
& Lab & 0,32 & 0,40 & 0,00 & \\
& BP & 0,64 & 0,39 & 0,50 & \\
& PP & 0,36 & 0,39 & 0,50 & \\
& LB & 0,55 & 0,42 & 0,50 & \\
& Cpo & 0,36 & 0,39 & 0,50 & \\
& AB & 0,59 & 0,49 & 1,00 &
\end{tabular}

Legenda: CT: Cubos em Torre; CP: Cubos em Ponte; Agu: Agulha; Nó; Lab: labirinto; BP: Bolinha de Papel; PP: Ponta do polegar; LB: Lançar uma Bola; Cpo: Círculo com polegar; AB: Agarrar uma Bola.

*significância entre as variáveis de interesse.

Teste de Friedman; valor de p: 0,05. 
Tabela 3 - Comparação entre os subtestes de função motora fina da escala de desenvolvimento motor

\begin{tabular}{|c|c|c|c|c|c|}
\hline \multirow{2}{*}{ Subtestes } & \multicolumn{2}{|c|}{ Grupo } & \multirow{2}{*}{ Subtestes } & \multicolumn{2}{|c|}{ Grupo } \\
\hline & I & II & & I & II \\
\hline $\mathrm{CP}-\mathrm{CT}$ & $>0,999$ & $>0,999$ & $A B-A g u$ & 0,102 & $0,034^{*}$ \\
\hline $\mathrm{Agu}-\mathrm{CT}$ & $>0,999$ & $>0,999$ & lab - Nó & $0,003^{*}$ & $0,021^{*}$ \\
\hline Nó - CT & 0,317 & 0,157 & BP - Nó & $0,023^{*}$ & 0,157 \\
\hline $\mathrm{lab}-\mathrm{CT}$ & $0,002^{*}$ & $0,006^{*}$ & PP - Nó & $0,009^{*}$ & 0,059 \\
\hline$B P-C T$ & $0,014^{*}$ & $0,023^{*}$ & LB - Nó & $0,014^{*}$ & 0,301 \\
\hline$P P-C T$ & $0,006^{*}$ & $0,006^{*}$ & Cpo - Nó & $0,009^{*}$ & $0,026^{*}$ \\
\hline $\mathrm{LB}-\mathrm{CT}$ & $0,008^{*}$ & $0,015^{\star}$ & AB - Nó & 0,180 & 0,258 \\
\hline Cpo - CT & $0,006^{*}$ & $0,006^{*}$ & $B P-l a b$ & 0,114 & 0,070 \\
\hline$A B-C T$ & 0,102 & $0,034^{*}$ & $P P-l a b$ & 0,112 & 0,773 \\
\hline $\mathrm{Agu}-\mathrm{CP}$ & $>0,999$ & $>0,999$ & LB - lab & 0,194 & 0,194 \\
\hline Nó - CP & 0,317 & 0,157 & Cpo - lab & $0,020^{*}$ & 0,763 \\
\hline$l a b-C P$ & $0,002^{*}$ & $0,006^{*}$ & $A B-l a b$ & $0,007^{*}$ & 0,084 \\
\hline $\mathrm{BP}-\mathrm{CP}$ & $0,014^{*}$ & $0,023^{*}$ & $P P-B P$ & 0,655 & 0,058 \\
\hline$P P-C P$ & $0,006^{*}$ & $0,006^{*}$ & LB - BP & 0,589 & 0,608 \\
\hline $\mathrm{LB}-\mathrm{CP}$ & $0,008^{*}$ & $0,015^{\star}$ & Cpo - BP & 0,763 & 0,058 \\
\hline Cpo - CP & $0,006^{*}$ & $0,006^{*}$ & $A B-B P$ & 0,068 & 0,792 \\
\hline$A B-C P$ & 0,102 & $0,034^{*}$ & LB - PP & 0,705 & 0,157 \\
\hline Nó - Agu & 0,317 & 0,157 & Cpo-PP & $>0,999$ & $>0,999$ \\
\hline $\mathrm{lab}-\mathrm{Agu}$ & $0,002^{*}$ & $0,006^{*}$ & $A B-P P$ & $0,033^{*}$ & 0,248 \\
\hline $\mathrm{BP}-\mathrm{Agu}$ & $0,014^{*}$ & $0,023^{*}$ & Cpo - LB & 0,705 & 0,206 \\
\hline $\mathrm{PP}-\mathrm{Agu}$ & $0,006^{*}$ & $0,006^{*}$ & $A B-L B$ & $0,024^{*}$ & 0,705 \\
\hline LB - Agu & $0,008^{*}$ & $0,015^{\star}$ & AB - Cpo & $0,011^{*}$ & 0,129 \\
\hline Cpo-Agu & $0,006^{*}$ & $0,006^{*}$ & & & \\
\hline
\end{tabular}

Legenda: CT: Cubos em Torre; CP: Cubos em Ponte; Agu: Agulha; Nó; Lab: labirinto; BP: Bolinha de Papel; PP: Ponta do polegar; LB: Lançar uma Bola; Cpo: Círculo com polegar; AB: Agarrar uma Bola.

*diferenças estatisticamente significante quando as variáveis comparadas par a par.

Teste dos Postos Sinalizados de Wilcoxon; valor de p: 0,05

com labirinto (Lab), bolinha de papel (BP), ponta do polegar (PP), lançar uma bola (LB) e círculo com o polegar (Cpo). Apresentaram desempenho superior na prova da agulha (Agu) se comparado com labirinto (Lab), bolinha de papel (BP), ponta do polegar (PP), lançar uma bola (LB) e círculo com o polegar (Cpo). Apresentaram desempenho superior na prova do nó (Nó) se comparado com labirinto (Lab), bolinha e papel (BP), ponta do polegar (PP), lançar uma bola (LB) e círculo com o polegar (Cpo). Apresentaram desempenho superior na prova do labirinto (Lab) se comparado a círculo com polegar (Cpo) e agarrar uma bola (AB). Apresentaram desempenho superior na prova de ponta do polegar
(PP) se comparado com agarrar uma bola $(A B)$. E apresentaram desempenho superior em lançar uma bola (LB) e na prova círculo com polegar (Cpo) em relação a agarrar uma bola $(A B)$.

Os escolares do Gll apresentaram desempenho superior em construção de cubos em torre (CT) se comparado a labirinto (Lab), bolinha de papel (BP), ponta do polegar (PP), lançar uma bola (LB), círculo com o polegar (Cpo) e agarrar uma bola (AB). Apresentaram desempenho superior na construção de cubos em ponte (CP) se comparado a labirinto (Lab), bolinha de papel (BP), ponta do polegar (PP), lançar uma bola (LB), círculo com o polegar (Cpo) e agarrar uma bola $(\mathrm{AB})$. Apresentaram desempenho 
superior na prova da agulha (Agu) se comparado a labirinto (Lab), bolinha de papel (BP), ponta do polegar (PP), lançar uma bola (LB), círculo com o polegar (Cpo) e agarrar uma bola (AB). E apresentaram desempenho superior na prova do nó (Nó) se comparado a labirinto (Lab) e círculo com polegar (Cpo).

Apesar de os escolares com dislexia e TDAH apresentarem dificuldades na realização das atividades motoras finas, os escolares com TDAH apresentaram maior média de acertos para execução de tarefas motoras finas do que os escolares com dislexia, como observado nas atividades de nó, labirinto, ponta do polegar, lançar uma bola e círculo com polegar.

A tabela 4 apresenta a comparação intra-grupo da idade cronológica e idade motora fina dos escolares do Gl e Gll. Com a aplicação do Teste dos Postos Sinalizados de Wilcoxon, verificou-se diferença estatisticamente significante para ambos os grupos, revelando que a idade motora fina tanto dos escolares com dislexia como com TDAH é inferior à idade cronológica.

Tabela 4 - Distribuição da média, desvio padrão e valor de p dos grupos GI e Gll para comparação entre a idade motora fina e idade cronológica

\begin{tabular}{cccccc}
\hline Grupo & Subtestes & Média & Desvio-padrão & Mediana & Valor de $\mathbf{p}$ \\
\hline \multirow{2}{*}{ I } & IC & 9,54 & 1,94 & 9,67 & \multirow{2}{*}{$0,003^{*}$} \\
& IMF & 4,91 & 0,54 & 5,00 & \\
\hline \multirow{2}{*}{ II } & IC & 9,96 & 1,08 & 10,00 & $0,003^{*}$ \\
& IMF & 5,55 & 1,25 & 5,00 & \\
\hline
\end{tabular}

Legenda: IC: Idade Cronológica; IMF: Idade Motora Fina.

*significância entre ambas as idades.

Teste dos Postos Sinalizados de Wilcoxon; valor de p: 0,05

Quando foram correlacionados os resultados dos subtestes com o número de escolares deste estudo, verificou-se que ocorreu correlação estatisticamente significante, a qual foi positiva, indicando que quanto menor o desempenho em pontuação dos escolares na atividade de fazer bolinha

Tabela 5 - correlação entre os subtestes da função motora fina para os escolares deste estudo

\begin{tabular}{|c|c|c|c|c|c|c|c|c|c|c|c|}
\hline Variável & Estatística & CT & $\mathrm{CP}$ & Agu & Nó & lab & BP & PP & LB & Cpo & $A B$ \\
\hline \multirow{2}{*}{$\mathrm{BP}$} & $\begin{array}{l}\text { Coef. } \\
\text { Correl. }\end{array}$ & - & - & - & 0,356 & 0,042 & 1,000 & 0,553 & 0,213 & 0,347 & 0,255 \\
\hline & Sig. (p) & - & - & - & 0,104 & 0,853 & - & $0,008^{*}$ & 0,341 & 0,114 & 0,253 \\
\hline \multirow[t]{2}{*}{ PP } & $\begin{array}{l}\text { Coef. } \\
\text { Correl. }\end{array}$ & - & - & - & 0,090 & $-0,360$ & 0,553 & 1,000 & 0,480 & 0,488 & 0,244 \\
\hline & Sig. (p) & - & - & - & 0,690 & 0,100 & $0,008^{*}$ & - & $0,024^{*}$ & $0,021^{*}$ & 0,274 \\
\hline \multirow[t]{2}{*}{ LB } & $\begin{array}{l}\text { Coef. } \\
\text { Correl. }\end{array}$ & - & - & - & $-0,189$ & $-0,057$ & 0,213 & 0,480 & 1,000 & 0,458 & 0,487 \\
\hline & Sig. (p) & - & - & - & 0,399 & 0,800 & 0,341 & $0,024^{*}$ & - & $0,032^{*}$ & $0,022^{*}$ \\
\hline \multirow[t]{2}{*}{ Сpo } & $\begin{array}{l}\text { Coef. } \\
\text { Correl. }\end{array}$ & - & - & - & 0,237 & 0,165 & 0,347 & 0,488 & 0,458 & 1,000 & 0,562 \\
\hline & Sig. (p) & - & - & - & 0,289 & 0,464 & 0,114 & $0,021^{*}$ & $0,032^{*}$ & - & $0,006^{*}$ \\
\hline \multirow[t]{2}{*}{$A B$} & $\begin{array}{l}\text { Coef. } \\
\text { Correl. }\end{array}$ & - & - & - & 0,295 & 0,255 & 0,255 & 0,244 & 0,487 & 0,562 & 1,000 \\
\hline & Sig. (p) & - & - & - & 0,183 & 0,252 & 0,253 & 0,274 & $0,022^{*}$ & $0,006^{*}$ & - \\
\hline
\end{tabular}

Legenda: CT: Cubos em Torre; CP: Cubos em Ponte; Agu: Agulha; Nó; Lab: labirinto; BP: Bolinha de Papel; PP: Ponta do polegar; LB: Lançar uma Bola; Cpo: Círculo com polegar; AB: Agarrar uma Bola.

*significância na diferença entre ambos os grupos.

Análise de Correlação de Spearman; valor de p: 0,05. 
de papel (BP) menor o desempenho em pontuação na atividade ponta do polegar (PP); o mesmo ocorrendo entre a atividade ponta do polegar (PP) com as atividades do labirinto (Lab) e do círculo com o polegar (Cpo); entre a atividade de lançar uma bola (LB) com as atividades ponta do polegar (PP), círculo com polegar (Cpo) e agarrar uma bola (AB); e entre a atividade círculo do polegar (Cpo) com as atividades ponta do polegar (PP), lançar uma bola (LB) e agarrar uma bola (AB).

Esses achados indicam que tanto os escolares com dislexia como os escolares com TDAH apresentaram dificuldades em atividades de preensão e pressão de objetos e coordenação visuo-espacial, o que pode ser justificado pela idade motora fina aquém do esperado para idade e escolaridade.

A partir dos achados deste estudo, observou-se que conforme a classificação da Escala do Desenvolvimento Motor, $90 \%$ dos escolares com dislexia e dos escolares com TDAH apresentaram desenvolvimento motor fino muito inferior ao esperado para a idade e $10 \%$ dos escolares com dislexia apresentam desenvolvimento normal baixo ao esperado para a idade, e 10\% dos escolares com TDAH apresentaram desenvolvimento inferior ao esperado para a idade.

\section{DISCUSSÃO}

A coordenação motora fina é a função motora necessária para a realização de atividades que exijam destreza e os escolares com déficits motores geralmente têm dificuldade de adquirir habilidades motoras condizentes com a idade e acabam apresentando dificuldades nas tarefas funcionais diárias, no lazer e nas tarefas escolares ${ }^{3,15,16}$.

Com base nos dados obtidos, pôde-se ser observado que quando comparado os grupos deste estudo, tanto os escolares com dislexia quanto os escolares com TDAH apresentaram atraso no desenvolvimento da coordenação motora fina, corroborando os estudos descritos na literatura nacional ${ }^{14}$ e internacional ${ }^{8,31,32}$.

Nos resultados deste estudo, pôde-se verificar que tanto os escolares com dislexia como os escolares com TDAH apresentaram dificuldades na execução de atividades como: fazer bolinha de papel, ponta do polegar, círculo com o polegar, labirinto, lançar uma bola e agarrar uma bola. Entretanto, verificou-se que os escolares com TDAH apresentaram maior média de acerto para execução de tarefas motoras finas do que os escolares com dislexia, de acordo com a análise quantitativa de pontuação das provas.

Essas atividades cujos escolares deste estudo apresentaram dificuldades são descritas na literatura ${ }^{17,20,33,34}$ como aquelas que necessitam de movimentos de pinça tridigital, pinça polpa-polpa, oposição do polegar e movimentação do punho, além de graduação de força e pressão dos objetos e sincronização dos movimentos, exigindo alto grau de destreza motora, necessária para a aquisição do grafismo, além da integridade neuropsicológica necessária para a organização das informações exigidas para a execução dos movimentos finos.

Em geral, o TDC ${ }^{35,36}$ é caracterizado pelo comprometimento do desempenho motor, incluindo a coordenação motora fina, que se apresenta com idade motora inferior à idade cronológica, como observado nos escolares com dislexia e naqueles com TDAH deste estudo.

As alterações de função motora fina mostraramse presentes nestas populações, levando à reflexão sobre a possibilidade de ocorrência de TDC em comorbidade com a dislexia e com o TDAH, corroborando os estudos nacionais ${ }^{14} \mathrm{e}$ internacionais ${ }^{32,37}$, que descreveram que as alterações de função motora fina compõem o quadro de transtorno do desenvolvimento da coordenação (TDC), e que isso pode ser considerado uma comorbidade relacionada com a dislexia e o TDAH.

Como a avaliação motora é um elemento importante no exame de saúde geral de crianças, tornase necessário que os aspectos quantitativos e qualitativos da investigação dos movimentos motores finos e globais sejam investigados, uma vez que estes podem refletir a integridade e a maturidade do cérebro e podem, provavelmente, fornecer indícios de alterações no desenvolvimento motor, como os observados nos escolares com dislexia e TDAH descrito na literatura ${ }^{6,9,12,15}$.

Essas alterações motoras quando presentes no quadro de dislexia e no quadro de TDAH e não evidenciadas no processo diagnóstico fazem com que as condutas, tanto terapêuticas quanto psicoeducacionais em relação a esses escolares, sejam inadequadas para suas necessidades, podendo desencadear problemas de baixa autoestima, fracassos escolares e desmotivação para a aprendizagem ${ }^{15}$.

Portanto, em uma perspectiva diagnóstica, a determinação do perfil motor de escolares com dislexia e TDAH pode auxiliar na identificação não apenas da comorbidade destas condições com o TDC, como também na conduta terapêutica e educacional destes escolares que necessitam de enfoque clínico e educacional voltado para a minimização do impacto das manifestações comportamentais e cognitivo-linguísticas inerentes à dislexia e ao TDAH visando a uma melhor qualidade de vida social e acadêmica destes indivíduos. 
Entretanto, como este estudo apenas enfoca a coordenação motora fina em um número restrito de escolares, isso pode ser apenas considerado um indicador de alterações motoras que devem ser melhor avaliadas no contexto do desenvolvimento motor global para a investigação do TDC nesta população.

\section{CONCLUSÃO}

A partir dos resultados deste estudo, pode-se concluir que tanto os escolares com dislexia quanto os escolares com TDAH apresentaram atrasos na coordenação motora fina em relação à idade cronológica, revelando que $90 \%$ dos escolares com dislexia e dos escolares com TDAH apresentaram desenvolvimento motor fino muito inferior ao esperado para a idade e $10 \%$ apresentam desenvolvimento normal baixo ao esperado para a idade, demonstrando que os participantes desta pesquisa apresentam dificuldades em atividades que exijam destreza como a grafia e amarrar sapatos. Os achados idênticos entre os dois grupos se devem ao quadro de TDC que justificam seus desempenhos similares nas provas motoras finas realizadas.

Em relação à comparação do desempenho dos escolares do GI e Gll entre os subtestes de coordenação motora fina foi evidenciado que os escolares de ambos os grupos apresentam alterações em habilidades motoras finas que influenciam diretamente a aquisição do grafismo. Os achados de correlação indicaram que os escolares com dislexia e os escolares com TDAH apresentam dificuldades em atividades de preensão e pressão de objetos e coordenação visuo-espacial que podem ser justificadas pela idade motora fina aquém do esperado para idade e escolaridade, no entanto, o desempenho motor fino dos escolares com TDAH é superior ao desempenho dos escolares com dislexia em atividades que exijam destreza e precisão motora fina.

Assim, a partir dos resultados deste estudo, pôde-se concluir que os escolares com dislexia e os escolares com TDAH deste estudo apresentam alteração de função motora fina, se diferindo em seus desempenhos quanto a precisão e preensão motora fina, sendo que os escolares com TDAH apresentaram desempenho superior nestas habilidades motoras finas em comparação aos escolares com dislexia. Assim, estudos complementares estão sendo conduzidos pelos autores deste estudo para a partir do aumento do número de escolares com dislexia e TDAH poder verificar e comprovar se o perfil motor fino dos escolares encontrados neste estudo se assemelham ou se diferem de acordo com o quadro apresentado pelos escolares.

\section{ABSTRACT}

Purpose: to describe and compare the fine motor coordination performance of students with dyslexia and with Attention Deficit and Hyperactivity Disorder. Method: the study included 22 elementary school students of both genders, aged from 6 to 11-year old, divided into: GI: 11 students with Attention Deficit and Hyperactivity Disorder and GIl: 11 students with dyslexia. For the procedure, tests of fine motor skills from the Motor Development Scale were applied. Results: the results showed a statistically significant difference between the fine motor age and chronological age of the students at GI and Gll. According to the classification of the Motor Development Scale, $90 \%$ of students from GI and GII demonstrated fine motor development much lower than expected for their age, $10 \%$ of the students with dyslexia demonstrated low normal development as for the expected value according to age and $10 \%$ of students with Attention Deficit and Hyperactivity Disorder demonstrated inferior development than what is expect for their age. Conclusion: we concluded that both students with dyslexia and with ADHD in this study show a delay in fine motor coordination, demonstrating that the participants of this research have difficulties in tasks that require dexterity, characteristic condition of the coordination development disorder. Additional studies are being conducted by the authors of this study in order to ascertain and to check if the fine motor profiles of students in this study is similar or differ according to their condition.

KEYWORDS: Motor Skills; Dyslexia; Attention Deficit Disorder with Hyperactivity 


\section{REFERÊNCIAS}

1. Viholainen $\mathrm{H}$, Ahonen $\mathrm{T}$, Lyytinen $\mathrm{P}$, Cantell $M$, Tolvanen A, Lyytinen $H$. Development of early motor skills and language in children at risk of familial dyslexia. Dev Med Child Neurol. 2002; 44 (11): 761-9.

2. Diamond A. Close interrelation of motor development and cognitive development and of the cerebellum and prefrontal cortex. Child Dev. 2000; 71 (1):44-56.

3. Capellini AS, Souza AV. Avaliação da função motora fina, sensorial e perceptiva em escolares com dislexia. In: Sennye AL, Capovilla FC, Montiel, JM. Transtornos da aprendizagem da avaliação à reabilitação. São Paulo: Artes Médicas; 2008: 55-64.

4. Schirmer CR, Fontoura DR, Nunes ML. Language and learning disorder. J Pediatr. 2004; 80 (Suppl 2): 95-103.

5. Kolb B, Whishaw IQ. Neurociências do comportamento. Barueri: Manole; 2002.

6. Carboni-Román A, Del Río Grande D, Capilla A, Maestú $F$, Ortiz T. Bases neurobiológicas de las dificultades de aprendizaje. Rev Neurol. 2006; 42 (Suppl 2): S171-5.

7. Grizzle KL. Developmental Dyslexia. Pediatr Clin N Am. 2007; 54 (3): 507-23.

8. Mulas F, Etchepareborda MC, Díaz-Lucero A, Ruiz-Andrés R. El lenguaje y los trastornos del neurodesarrollo: revisión de las características clínicas Rev Neurol. 2006; 42 (Supl 2): S103-S9.

9. Soliva-Vila JC, Vilarroya-Oliver O. Aportaciones de la resonancia magnética estructural al esclarecimiento de la neurobiología del trastorno por déficit de atención/hiperactividad: hacia la identificación de un fenotipo neuroanatómico. Rev. Neurol. 2009; 48 (11): 592-8.

10. Magallón S, Narbona J. Detección y estudios específicos en el trastorno de aprendizaje procesal. Rev Neurol. 2009; 48 (Suppl 2): S71-S6.

11. Fliers E, Rommelse N, Vermeulen SHHM, Altink M, Buschgens CJM, Faraone SV, et al. Motor coordination problems in children and adolescents with ADHD rated by parents and teachers; effects of age and gender. J Neur Transm. 2008; 115 (2): 211-20.

12. Fliers E, Vermeulen S, Rijsdijk F, Altink M, Buschgens C, Rommelse N, et al. ADHD and Poor Motor Performance From a Family Genetic Perspective. J Am Acad Child Adolesc Psychiatry. 2009; 48(1): 25-34.

13. Capellini SA, Ferreira TL, Salgado CA, Ciasca $\mathrm{SM}$. Desempenho de escolares bons leitores, com dislexia e com transtorno do déficit de atenção e hiperatividade em nomeação automática rápida. Rev soc bras fonoaudiol. 2007; 12 (2): 114-9.

14. Toniolo CS, Santos LCA, Lourenceti MD, Padula, NAMR, Capellini SA. Caracterização do desempenho motor em escolares com transtorno do déficit de atenção com hiperatividade. Rev. Ass. Bras. Psicop. 2009; 79 (26): 33-40.

15. Trevisan JG, Copped AC, Capellini SA. Avaliação da função motora fina, sensorial e perceptiva em escolares com dificuldades de aprendizagem. Temas Desenvol. 2008; 16 (94):183-7.

16. Nicolson R, Fawcett, AJ. Dyslexia, dysgraphia, procedural learning and the cerebellum. Cortex 2009 doi:10.1016/j.cortex.2009.08.

17. Summers J, Larkin D, Dewey D. Activities of daily living in children with developmental coordination disorder: dressing, personal hygiene, and eating skills. Hum Mov Sc. 2008; 27 (2): 215-29.

18. Ciasca SM, Capellini AS, Tonelotto JMF. Distúrbios específicos de aprendizagem. In: Ciasca, SM. Distúrbio de aprendizagem: proposta de avaliação interdisciplinar. São Paulo: Casa do Psicólogo; 2003: 55-66.

19. GABBARD, C.; CAÇOLA, P. Los niños con trastorno del desarrollo de la coordinación tienen dificultad on la representación de las acciones. Rev Neurol. 2010; 50: 33-8.

20. Goez H, Zelnik N. Handedness in patients with developmental coordination disorder. J Child Neurol 2008; 23(2):151-4.

21. Rosenblum, S., Aloni, T. \& Josman, N. Relationships between handwriting performance and organizational abilities among children with and without dysgraphia: A preliminary study. Res Develop Disab. 2009, recuperado de doi:10.1016/j. ridd.2009.10.016

22. Flapper BCT, Howen S, Schoemaker MM. Fine motor skills and effects of methylphenidate in chikdren whith attention-deficit-hyperactivity disorder and developmental coordination disorder. Develop Med Child Neur. 2006, 48: 165-9.

23. Folio MR, Fewell RR. Peadoby Developmental Motor Scales. 2nd ed. Austin: PROED; 2000.

24. Scrutton D. Current and future uses of the Gross Motor Function Classification System. Dev Med Child Neurol. 2008; 50: 957.

25. Henderson SE, Sugden DA. Movement Assessment Battery for Children. 2nd ed. London: Psychological Corporation; 2007.

26. Beckung E. Development and validation of a measure of motor and sensory function in children with epilepsy. Pediatr Ther. 2000; 2: 24-35.

27. Fonseca V. Manual de observação psicomotora: significação neurobiológica dos fatores psicomotores. Porto Alegre: Artmed; 1995. 
28. Rosa Neto F. Manual de avaliação motora. Porto Alegre: Artmed; 2002.

29. Fundação de Economia e Estatística (FEE) [homepage na internet] Porto Alegre: 2003. [acesso em dez 2008]. Disponível em: http://www.fee.tche. br/sitefee/pt/content/estatisticas/pg_idese.php.

30. Capellini SA, Trevisan JG, Pavan JPM, Coppede A, Gonçalves, MPS, Ferreira ALB Dificuldades de aprendizagem: manual de orientação a professores quanto aos aspectos motores. v. 2. Marília - SP: Fundepe; 2007.

31. Meyer A, Sagvolden T. Fine motor skills in South African children with symptoms of ADHD: influence of subtype, gender, age, and hand dominance. Behav Brain Funct. 2006; 9: 2-33.

32. Rommelse NN, Altink, ME, Fliers, EA, Martin NC, Buschgens CJ, Hartman CA, et al. Comorbid Problems in ADHD: Degree of Association, Shared Endophenotypes, and Formation of Distinct Subtypes. Implications for a Future DSM. J Abnorm Child Psychol. 2009; 37 (6): 793-804.
33. Engel-Yeger B, Nagauker-Yanuv L, Rosenblum S. Handwriting performance, self-reports, and perceived self-efficacy among children with dysgraphia. Am J Occup Ther. 2009; 63 (2):182-92. 34. Kohlmeyer K. Avaliação dos componentes de desempenho. In: Neistadt M, Crepeau, EB. Terapia Ocupacional. 9a ed. Rio de Janeiro: Guanabara Koogan; 2003; 202-37.

35. GABBARD, C. Studying action representation in children via motor imagery. Brain and Cognition 2009, 71:234-9.

36. Vidarte JA, Ezquerro M, Giráldez MA. Perfil psicomotor de niños de 5 a 12 años diagnosticados clínicamente de trastorno por déficit de atención/ hiperactividad en Colombia. Rev Neurol. 2009; 49 (2): 69-75.

37. Dewey D, Camtell M, Crowford S. Motor and gestural performance in children with autism spectrum disorders, developmental coordination disorder and/or attention deficit hyperactivity disorder. J Internat Neuropsych Soc. 2007, 13, 246-56.
http://dx.doi.org/10.1590/S1516-18462011005000048

RECEBIDO EM: 14/05/2010

ACEITO EM: 20/12/2010

Endereço para correspondência:

Paola Matiko Martins Okuda

Rua Aviador Gomes Ribeiro, 23-50 apto. 26,

Jd. Brasil - Bauru-SP

CEP: 17011-067

E-mail: paolaokuda@yahoo.com.br 